\title{
Single cells and transposable element heterogeneity in stem cells and development
}

\author{
Andrew P. Hutchins ${ }^{1,2}$ (D)
}

\begin{abstract}
Recent innovations in single cell sequencing-based technologies are shining a light on the heterogeneity of cellular populations in unprecedented detail. However, several cellular aspects are currently underutilized in single cell studies. One aspect is the expression and activity of transposable elements (TEs). TEs are selfish sequences of DNA that can replicate, and have been wildly successful in colonizing genomes. However, most TEs are mutated, fragmentary and incapable of transposition, yet they are actively bound by multiple transcription factors, host complex patterns of chromatin modifications, and are expressed in mRNAs as part of the transcriptome in both normal and diseased states. The contribution of TEs to development and cellular function remains unclear, and the routine inclusion of TEs in single cell sequencing analyses will potentially lead to insight into stem cells,

development and human disease.
\end{abstract}

\section{Main text}

TEs are self-replicating sequences of DNA that have colonized nearly $50 \%$ of typical mammalian genomes, and take up more DNA sequence than the exons of coding genes. TEs that successfully duplicate during embryogenesis or in the germ cells can potentially enter the next generation. Consequently, TEs are particularly active during embryogenesis. However, over evolutionary time TEs lose their ability to replicate, through a mixture of sequence mutations, defective copying of TEs, and suppression by transcriptional and epigenetic silencing. Consequently, the vast majority of TEs in the human genome are not capable of transposition. Yet, despite being molecular fossils, there is a growing body of evidence that these fossil TEs are involved in normal developmental processes, including stem cells (Wang et al. 2020). TEs maintain complex patterns of chromatin

\footnotetext{
Correspondence: andrewh@sustech.edu.cn

'Shenzhen Key Laboratory of Gene Regulation and Systems Biology, Southern University of Science and Technology, Shenzhen 518055, China ${ }^{2}$ Department of Biology, School of Life Sciences, Southern University of Science and Technology, Shenzhen 518055, China
}

modifications, and can be transcribed into mRNAs and form parts of other coding or noncoding transcripts (Bourque et al. 2018) (Fig. 1a). These effects can have functional impacts on embryonic development. For example, knocking down mRNAs containing LINE L1 sequences leads to arrest at the embryonic 2-cell stage, as LINE L1-containing mRNAs work with Nucleolin, and Trim28/Kap1 to suppress Dux expression, and allow embryos to exit the 2-cell stage (Percharde et al. 2018). TE-containing RNAs are expressed in both a TE-type and a stage-specific manner during embryonic development. For example, in mouse and human embryogenesis each embryonic stage has a distinct pattern of TE expression (Goke et al. 2015; Wang et al. 2020). These patterns can be recapitulated in mouse pluripotent stem cells (PSCs). Mouse PSCs typically resemble the early epiblast in both gene and TE expression, but a small percentage of cells in mouse PSC cultures express a mouse-specific endogenous retrovirus (ERV), MERVL, which is also specifically expressed in 2-cell stage mouse embryos. Intriguingly, MERVL expressing cells have some totipotent properties, and gene expression 


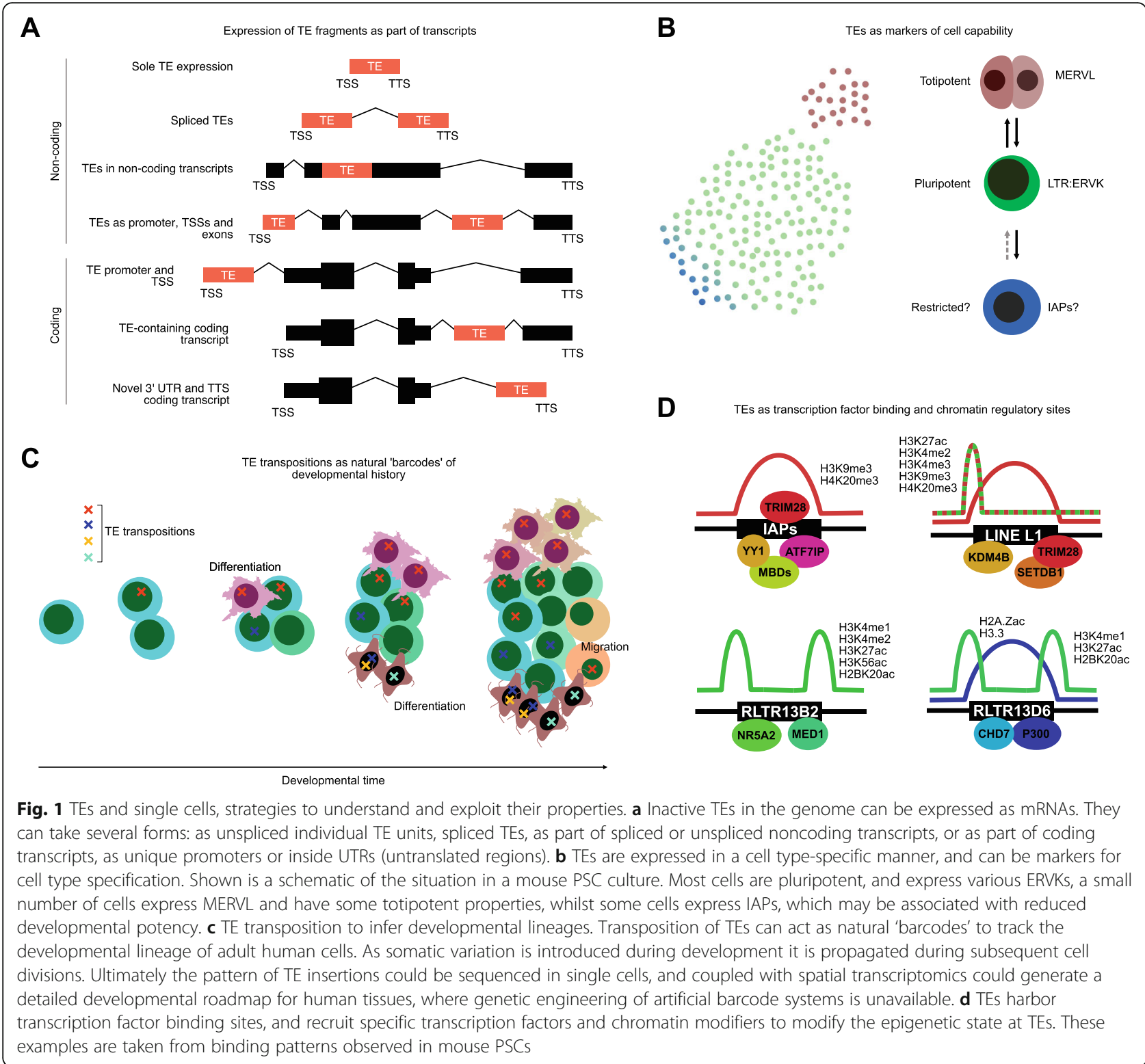

reminiscent of 2-cell stage embryos (Macfarlan et al. 2012) (Fig. 1b), and there are indications that MERVL mRNAs are functionally important in entry and exit from the 2-cell embryonic stage. TE expression can act as a molecular characteristic to define different states of human PSCs. For example, the human-specific HERVH is expressed in primed hPSCs that resemble the early epiblast, whilst the SVA family of TEs are expressed in naive hPSCs that resemble the inner cell mass (Theunissen et al. 2016). TEs may thus be a useful marker to explore sub-cell types with enhanced or restricted capabilities within cell cultures (Fig. 1b). TEs are also widely expressed in post implantation tissues, and two recent studies explored the expression patterns of TEs in mouse gastrulation using single cell RNA-seq data (He et al. 2021; Shao and Wang 2021). Both studies observed TE-type specific expression restricted to developmental lineages, and TE expression had especially complex patterns in extraembryonic tissues.

Whilst the vast majority of TEs are inactive, a few families of TEs are active in humans and mice. These 'hot' TEs retain the ability to transpose, and can introduce variance into the genome. The LINE L1 family is active in humans, and during embryogenesis several TE duplications are potentially introduced into single cells of the germ line, resulting in mosaic germ cells with novel TE insertions (Faulkner and Billon 2018). However, extraembryonic and somatic tissues are also sites of TE activity (Chuong 2018), and potentially transposition (Faulkner and Billon 2018). In humans, LINE L1s are 
particularly active in the extraembryonic and possibly gastrulating embryonic tissues, and LINEs are also active in the developing brain. When LINE L1s duplicate during brain development they are passed to their progeny, leading to mosaicism which could be used to trace the history of cell division and so the developmental lineage of the brain (Fig. 1c). An ingenious study took advantage of the somatic mosaicism of the LINE L1-family of TEs in the human brain, although limitations in technology at the time made it possible to look at only a small number of neurons (Evrony et al. 2015). However, innovations in spatial single cell RNA/DNA-seq, which preserves the location of a cell in its tissue, coupled to sequencing of novel retrotransposition events, could lead to a detailed developmental roadmap of the human brain. Whilst not currently feasible in a complete adult organism at the single cell level, technological innovations will eventually make this a viable strategy.

Nonetheless, the analysis of TEs is fraught with difficulty both for measuring TE transcript expression and novel TE insertions. Indeed, estimates of transposition rates in neuronal cells range widely from 0.04 per cell to 13.7 per cell (Faulkner and Billon 2018). The large range is due to problems in accurately sequencing novel insertions in the genome. Similar issues trouble the measurement of inactive TE sequence fragments spliced into transcripts (Babarinde et al. 2019). A common solution is to combine all TE copies of the same type into a single 'meta-element' that represents the activity across the genome. This approach can be helpful in single cell analysis, where mapping of short reads to TEs is difficult, and data sparsity remains a challenge. Nonetheless, combining TEs into a single meta-element sacrifices important TE information at specific genomic loci, which could be exploited to understand biological phenomena, such as TE functions at specific genes or regulatory regions. However, the uncertainties in identifying the precise genomic locus of a TE sequence in the DNA has hampered the discovery of simple relationships between a $\mathrm{TE}$ and a gene, and may explain the relative paucity of these specific relationships in the literature. An interesting study utilized transcript assembly to improve this aspect in single cell expression analysis (Shao and Wang 2021). In that study TEs were first assembled into transcripts using bulk short-read RNA-seq, and then the single cell RNA-seq data was mapped to the assembled transcripts. Their work can potentially place expressed TEs in their genomic context, which can unlock important information. However, assembling transcripts together from short reads is difficult to do accurately, even in species with robust genome and transcriptome annotations (Babarinde et al. 2019). A possible solution involves the application of long-read and single-molecule sequencing technologies to assemble full-length $\mathrm{TE}$ containing transcripts in single cells. A recent study exploited both short and long-reads to identify splicing patterns in single cells of mouse embryonic brain, although TEs were not addressed (Lebrigand et al. 2020). One complication however, is that long and short reads have both advantages and disadvantages: short reads have excellent dynamic range, but are poor at assembling transcripts, whilst, conversely, long reads are excellent at assembling transcripts, but have weak dynamic range, and can detect extremely rare, possibly spurious transcripts. Ultimately, some combination of long and short reads applied to sc-RNA-seq will be a powerful technique to exploit information from TE sequences in mRNAs.

When TEs are transpositionally active, they compete with the cell transcriptional machinery, and often contain transcription factor (TF) binding sites that the TE exploits to promote their own transcription and so transposition. Hence TEs can and do act as promoters and transcription start sites. In pluripotent stem cells the long terminal repeats (LTRs) from several ERV families act as pluripotent-specific transcription start sites (Fort et al. 2014). TEs thus exploit the endogenous transcriptional machinery to promote their own expression by containing TF binding sites for pluripotency TFs (Wang et al. 2020). During evolution, as TEs transpose, they shuffle the enhancer elements controlling pluripotency genes. This helps explain the dramatic differences in genome binding sites of two pluripotency TFs, OCT4 and NANOG, in mouse and human PSCs. Despite ostensibly performing the same function and regulating similar sets of genes, OCT4 and NANOG binding sites are substantially different between mouse and human PSCs, most likely due to the activity of TEs (Kunarso et al. 2010). This property of TEs can also help explain why TEs are expressed in somatic cells. Each TF in a family can bind a similar DNA sequence, yet different members of the same TF family can be active in widely divergent cell types and tissues, hence a TF that regulates a TE during embryogenesis may have a corresponding family member in somatic tissues that can activate the same TE. This may help explain the surprising widespread expression and activity of TEs even in somatic tissues, particularly the brain and the immune system (Faulkner and Billon 2018; He et al. 2021). A consequence of TFs binding to TEs is the presence of TE-type specific chromatin modifications ( $\mathrm{He}$ et al. 2019) (Fig. 1d). Some TE types are silenced by histone methylations, whilst others have active histone acetylation, and yet further TEs are bivalently marked with by both methylation and acetylation. Almost certainly divergent and combinatorial patterns of TFs binding to TEs is driving this effect. However, much remains to be discovered, as all of the above analysis was performed in 
pooled cells, meaning that TF and chromatin behavior in single cells at TEs remains unclear. Innovations in single cell methods that probe the epigenetic state (e.g. single cell chromatin accessibility or DNA methylation) may lead to insight into TF binding to TE sequences, and the consequences for regulation of the epigenome.

Ultimately, TEs are an integral part of the transcriptional output of cells, are major sites of chromatin regulation, and contribute to cell type heterogeneity in unclear ways. An Improved understanding of TE activity in single cells, as transcribed units, chromatin elements, and transposition events, will lead to insight into cellular function.

Author's contributions

The author read and approved the final manuscript.

\section{Funding}

This work was supported by National Natural Science Foundation of China (31970589) and the Shenzhen Innovation Committee of Science and Technology grants (ZDSYS20200811144002008).

\section{Declarations}

\section{Competing interests}

A.P.H. is a member of the Editorial Board for Cell Regeneration, and was not involved in the journal's review of, or decisions related to, this manuscript.

Received: 2 April 2021 Accepted: 30 April 2021

Published online: 06 July 2021

\section{References}

Babarinde IA, Li Y, Hutchins AP. Computational methods for mapping, assembly and quantification for coding and non-coding transcripts. Comput Struct Biotechnol J. 2019;17:628-37. https://doi.org/10.1016/j.csbj.2019.04.012.

Bourque G, Burns KH, Gehring M, Gorbunova V, Seluanov A, Hammell M, et al. Ten things you should know about transposable elements. Genome Biol. 2018;19(1):199. https://doi.org/10.1186/s13059-018-1577-z.

Chuong EB. The placenta goes viral: retroviruses control gene expression in pregnancy. PLoS Biol. 2018;16(10):e3000028. https://doi.org/10.1371/journal. pbio.3000028.

Evrony GD, Lee E, Mehta BK, Benjamini Y, Johnson RM, Cai X, et al. Cell lineage analysis in human brain using endogenous retroelements. Neuron. 2015; 85(1):49-59. https://doi.org/10.1016/j.neuron.2014.12.028.

Faulkner GJ, Billon V. L1 retrotransposition in the soma: a field jumping ahead. Mob DNA. 2018;9(1):22. https://doi.org/10.1186/s13100-018-0128-1.

Fort A, Hashimoto K, Yamada D, Salimullah M, Keya CA, Saxena A, et al. Deep transcriptome profiling of mammalian stem cells supports a regulatory role for retrotransposons in pluripotency maintenance. Nat Genet. 2014;46(6):55866. https://doi.org/10.1038/ng.2965.

Goke J, Lu X, Chan YS, Ng HH, Ly LH, Sachs F, et al. Dynamic transcription of distinct classes of endogenous retroviral elements marks specific populations of early human embryonic cells. Cell Stem Cell. 2015;16(2):135-41. https://doi. org/10.1016/.stem.2015.01.005.

He J, Babarinde IA, Sun L, Xu S, Chen R, Shi J, et al. Identifying transposable element expression dynamics and heterogeneity during development at the single-cell level with a processing pipeline scTE. Nat Commun. 2021;12(1): 1456. https://doi.org/10.1038/s41467-021-21808-x.

He J, Fu X, Zhang M, He F, Li W, Abdul MM, et al. Transposable elements are regulated by context-specific patterns of chromatin marks in mouse embryonic stem cells. Nat Commun. 2019;10(1):34. https://doi.org/10.1038/ s41467-018-08006-y.

Kunarso G, Chia NY, Jeyakani J, Hwang C, Lu X, Chan YS, et al. Transposable elements have rewired the core regulatory network of human embryonic stem cells. Nat Genet. 2010;42(7):631-4. https://doi.org/10.1038/ng.600.
Lebrigand K, Magnone V, Barbry P, Waldmann R. High throughput error corrected Nanopore single cell transcriptome sequencing. Nat Commun. 2020;11(1):4025. https://doi.org/10.1038/s41467-020-17800-6.

Macfarlan TS, Gifford WD, Driscoll S, Lettieri K, Rowe HM, Bonanomi D, et al. Embryonic stem cell potency fluctuates with endogenous retrovirus activity. Nature. 2012;487(7405):57-63. https://doi.org/10.1038/nature11244.

Percharde M, Lin CJ, Yin Y, Guan J, Peixoto GA, Bulut-Karslioglu A, et al. A LINE1Nucleolin partnership regulates early development and ESC identity. Cell. 2018;174(391-405):e319.

Shao W, Wang T. Transcript assembly improves expression quantification of transposable elements in single-cell RNA-seq data. Genome Res. 2021;31(1): 88-100. https://doi.org/10.1101/gr.265173.120.

Theunissen TW, Friedli M, He Y, Planet E, O'Neil RC, Markoulaki S, et al. Molecular criteria for defining the naive human pluripotent state. Cell Stem Cell. 2016; 19(4):502-15. https://doi.org/10.1016/..stem.2016.06.011.

Wang J, Huang J, Shi G. Retrotransposons in pluripotent stem cells. Cell Regen. 2020;9:4

\section{Submit your manuscript to a SpringerOpen ${ }^{\circ}$ journal and benefit from:}

- Convenient online submission

- Rigorous peer review

- Open access: articles freely available online

High visibility within the field

- Retaining the copyright to your article

Submit your next manuscript at $\boldsymbol{\nabla}$ springeropen.com 